\title{
Reinforcer and ratio requirement effects in concurrent fixed-interval fixed-ratio schedules*
}

\author{
KEITH A. WOOD and RICHARD D. WILLIS \\ University of Florida, Gainesville, Florida 32611
}

\begin{abstract}
The fixed-ratio requirement was varied in concurrent fixed-interval fixed-ratio schedules. Fixed-ratio responding was reinforced by food. In different phases, fixed-interval responding was reinforced by food and water. There was an inverted U relationship between the ratio requirement and ratio response rates. There was a direct relation between the ratio requirement and interval response rates when both responses were reinforced with food, but only minimal relational effects when the reinforcers were different. The role of reinforcers in concurrent schedules merits detailed study.
\end{abstract}

LaBounty and Reynolds (1973) described the performance of pigeons on concurrent fixed-interval fixed-ration (conc FI FR) schedules. Pecks on one key produced grain according to an FI 4-min schedule. Timed from a reinforcement or that key, the first peck after 4 min produced grain. Every nth peck on a second (FR) key produced grain. Increasing the FR requirement decreased FR response rates and increased FI response rates.

Catania (1966) suggested that performance on concurrent schedules may depend on the reinforcers used. The data reported here support Catania's idea. We compared performance on conc FI(food) FR(food) schedules with performance on conc FI(water) FR(food) schedules.

\section{METHOD}

\section{Subjects}

Two male experimentally naive Long-Evans descent hooded rats approximately 140 days old were deprived of both food and water. Daily food and water allotments were adjusted for each $S$ in order to maintain stable body weights. Both rats were housed individually in a light-dark cycle controlled colony room and were deprived of both food and water. The temperature was maintained at $70 \pm 2^{\circ} \mathrm{F}\left(21.1 \pm 1.1^{\circ} \mathrm{C}\right)$.

\section{Apparatus}

Two operant chambers each contained two retractable levers, $3.0 \mathrm{~cm}$ above the grid floor and $14.0 \mathrm{~cm}$ apart. The required response force was approximately $0.20 \mathrm{~N}$. A water dipper was $8.0 \mathrm{~cm}$ to the right of the left lever. The water reinforcer was 0.1-cc tap water, available for $2.5 \mathrm{sec}$ and accompanined by a 2.5 -sec clicker. A food magazine was $4.0 \mathrm{~cm}$ to the left of the right lever. The food reinforcer was one 0.045-g Noyes standard formula pellet, accompanied by a 2.5 -sec tone. Masking noise was continuously present except for $30 \mathrm{msec}$ following a response: The resulting "pop" served as a response feedback.

*The author wishes to thank Calvin $\mathrm{K}$. Adams for sponsoring this paper. This research and the writing of this report was supported in part by Research Grant GB-28716X1 from the National Science Foundation to R. D. Willis and C. K. Adams and in part by the Department of Psychology, the College of Arts and Sciences, and the Graduate School at the University of Florida. Reprints may be obtained from Keith A. Wood, Department of Psychology, University of Florida, Gainesville, Florida 32611.
Dim general illumination was provied by a 7.5-W lamp. Each chamber was located in a sound- and light-attenuating box. Standard electromechanical scheduling and recording equipment was located in an adjacent room.

\section{Procedure}

The rats were adapted to the food- and water-deprivation regimen and magazine trained. Then responding was reinforced on conc FI(water) FR(food) schedules, and the FR(food) requirment was varied. Then the FI reinforcer was changed to food, and the FR(food) requirement was varied.

Magazine training was conducted with the levers retracted. After a $S$ rapidly approached the water dipper following clicker onset and rapidly approached the food magazine following tone onset, both levers were introduced. Early in pretraining, the levers alternated until the left lever controlled responding appropriate to the FI(water) schedule, and the right lever controlled responding appropriate to the FR(food). schedule. Responding was then developed on a conc FI 4-min $\mathrm{LH}$ 11-sec(water) FR 10(food) schedule. Specifically, both levers were present. Left lever, FI responses were reinforced with water. Right lever, FR responses were reinforced with food. To aid the development of FI schedule control, a rat had a brief time (limited hold, or $\mathrm{LH}$ ) in which to respond, receive water, and restart the FI timer. In some aspects, the LH could act like a changeover delay in reducing the probability of superstitious chaining. At least 140 pretraining sessions preceded collecting the data reported here.

Daily sessions ended with the first water delivery (or the end of an LH) approximately " 90 min after the levers were inserted. Purina chow and water were provided after each session to maintain the same deprivations and stable body weights (less than $2 \mathrm{~g}$ difference per week). Rat 19 received approximately $18.5 \mathrm{~g}$ of - food and $17.5 \mathrm{~g}$ of water per day; Rat 25 received approximately $17 \mathrm{~g}$ of food and $22 \mathrm{~g}$ of water per day. These amounts of food and water maintained responding on both levers. When deprivation levels were different, either body weights gradually changed or responding would predominate on one lever (Willis, "Van Hartesveldt, Loken, \& Hall, in press). Fixed-interval responding on the left lever was reinforced with water. Concurrently, FR responding on the right lever was reinforced with food. The ratio requirement was increased by a factor of 1.2 for each session $(10,12,14,17,20,24,29, \ldots)$.

To compare responding on conc FI(water) FR(food) with responding on conc FI(food) FR(food), we used the ascending series with the same requirements as those used when the FI was reinforced with water. In this second phase, water was continuously available except in the chamber. The daily amount of food was decreased. Rat 19 received $12 \mathrm{~g}$ per day; Rat 25 , 

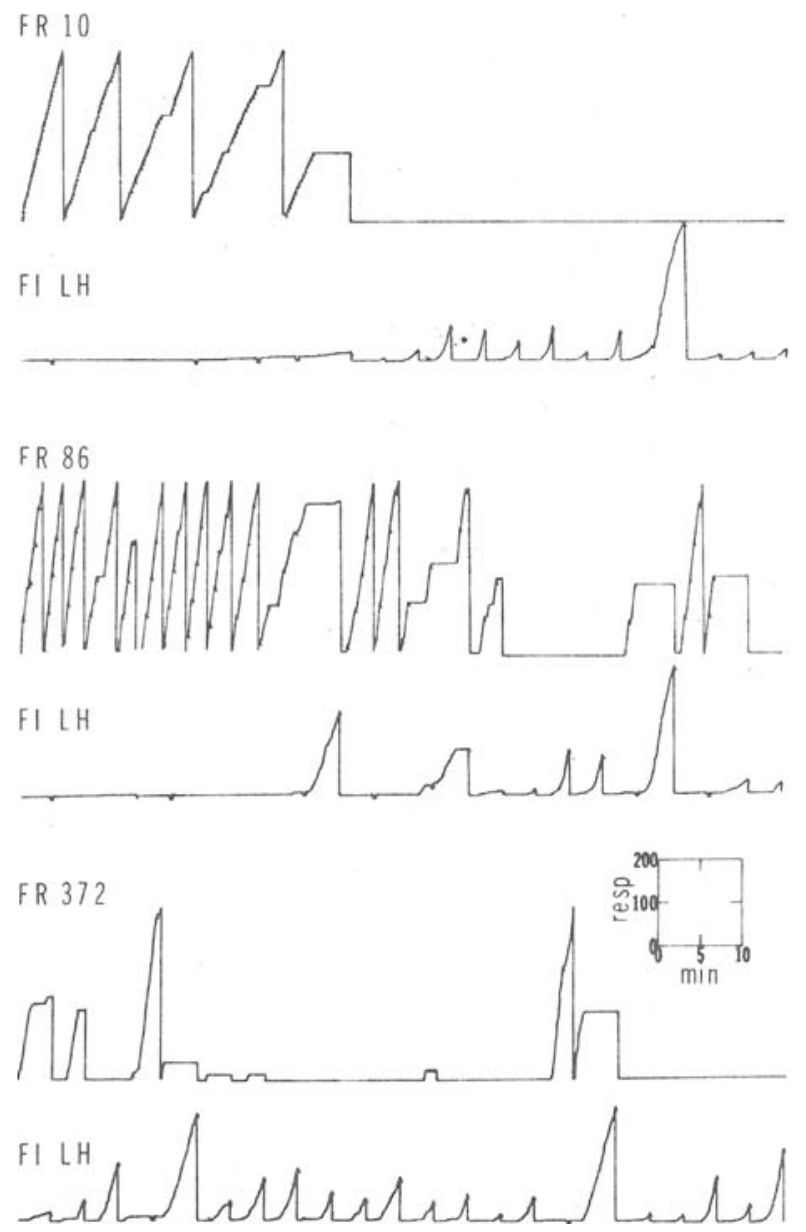

Fig. 1. Cumulative records of rates and patterns of responding by Rat 25 on conc FI(water) FR(food) at FR requirements of 10, 86, and 372. Paper drive motors ran throughout sessions. The top panel tracing is FR(food) responding; the bottom panel tracing is FI(water) responding. Cumulating pens reset after $\mathbf{4 0 0}$ responses and when ever water was delivered. The FI cumulating pen was displaced downward during the limited hold.

$13 \mathrm{~g}$ per day. Sessions were ended after the daily food amount was earned or approximately $90 \mathrm{~min}$.

The ascending series was used to investigate behavior to a wide range of ratio requirements while holding the FI requirement the same. The ascending series ended on the session prior to the one the $\mathrm{E}$ felt would totally eliminate food responding (typically, when the rat earned less than 10 food pellets in a session). A descending series was also employed in which the Ss experienced the same ratio requirements in reverse order. If the S's FR(food) response rate consistently decreased during the initial decreases of the ratio requirement, the descending series was voided and responding reestablished on the conc FI 4 min FR 10 schedule.

\section{RESULTS}

Cumulative records of typical performance to conc FI(water) FR(food) are presented in Fig. 1 (Rat 25). Performance on these three ratio requirements for Rat 25 were typical of Rat 19 also. Schedule-appropriate responding was maintained on both schedules over the full range of ratio requirements studied, with the Ss never responding exclusively on one lever. At low ratio requirements (FR 10), all FR(food) reinforcers were usually earned before the first FI(water) reinforcer was earned. At intermediate ratio requirements (FR 86), most of the FR(food) reinforcers were earned before the first FI(water) reinforcer. At high ratio requirements (FR 372), the first FR(food) reinforcer was earned between FI(water) reinforcers. The pattern of responding was a function of the ratio requirement: The higher the ratio requirement, the higher the probability the $\mathrm{S}$ would respond first in the FI component.

Cumulative records of conc FI(food) FR(food) (not shown) closely resembled those published by LaBounty

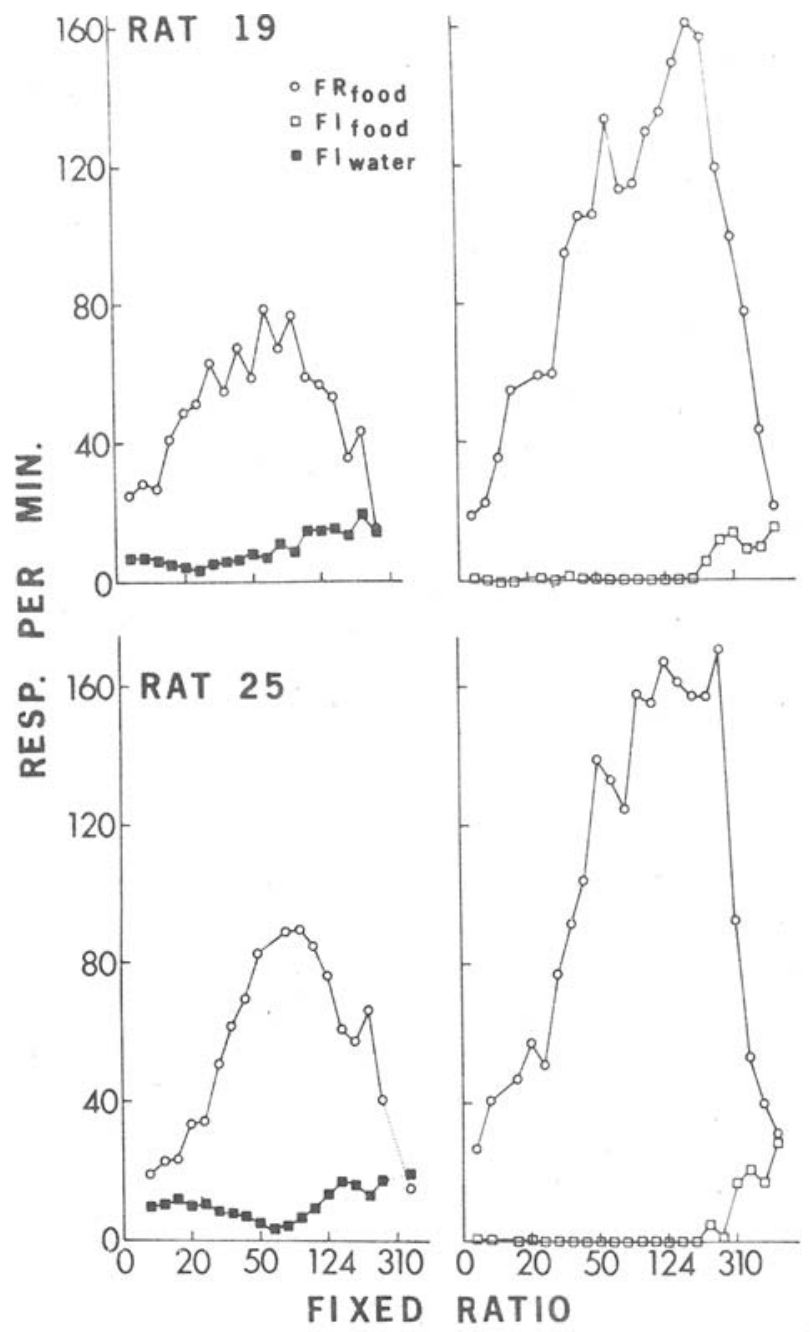

Fig. 2. Rates of responding for Rat 19 (top) and Rat 25 (bottom) as a function of the FR requirement. Squar es represent FI response rates; circles, FR response rates. Filled squares represnet FI(water) rates; open symbols represent food reinforced responding. Note the $\log$ scale for the fixed-ratio values. 
and Reynolds (1973). Absolute response rates for the ascending series are presented in Fig. 2. The number of responses was divided by the total session duration. The FR(food) response rates (open circles) have an inverted $\mathrm{U}$ shape function whether the concurrent FI responding was reinforced with food or water. Highest FR(food) response rates were reached when FI responding was reinforced with food. Fixed-interval(water) response rates (closed squares) were generally low at the low FR requirements, but gradually increased with the higher FR requirements. Fixed-interval(food) response rates (open squares) were extremely low at the low and intermediate FR requirements, but increased abruptly to some asymtope level at the high FR requirements.

\section{DISCUSSION}

The present results indicate that the reinforcers used in conc FR FI schedules substantially influence the effects of the FR requirment on response rates. That $\mathrm{FR}$ response rates under conc FI(water) FR(food) conditions were consistently lower than FR rates under conc FI(food) FR(food) conditions probably reflect the deprivation procedures used.

The greatest effect of different reinforcers was seen on FI responding. The relatively stable, gradual increase under conc FI(water) FR(food) conditions (Phase I) differs from those under conc FI(food) FR(food) conditions (LaBounty \& Reynolds, 1973; the present experiment Phase II). Fixed-interval response rates in Phase II were consistently lower than those of Phase I for the low and intermediate ratio requirements. When responding on both schedules was reinforced with food, FR reinforcement rate seemed to predict the maintenance of asymtopic FI responding. When water reinforced FI responding, no such reinforcement rate effect was seen. Further studies should consider schedule, stimulus, and schedule value effects in their investigations.

The present data merit systematic replications with other schedules and reinforcers. Catania (1973) pointed out that very little research has been done with concurrent schedules in which the reinforcers were different and that such studies should consider motivational interactions between the reinforcers used. We used rats as Ss and food and water reinforcers because so much is known about about relations between eating and drinking in rats (e.g., Bolles, 1967; Code, 1967). It is possible that the present finding on conc FI(water) FR(food) depend on the levels of concurrent deprivations of food and water. Effects of varying deprivation levels might differ from effects of changing schedule parameters. Alternatively, the effects could be complementary. Substantial data will be needed to resolve these important issues.

\section{REFERENCES}

Bolles, R. C. Theory of motivation. New York: Harper and Row, 1967.

Catania, A. C. Concurrent operants. In W. K. Honig (Ed.), Operant behavior: Areas of research and application. New York: Appleton-Century-Crofts, 1966, Pp. 213-270.

Catania, A.C. Self-inhibiting ef fects of reinforcement. Journal of the Experimental Analysis of Behavior, 1973, 19, 517-526.

Code, C. F. (Ed.) Handbook of physiology: Section 6: Alimentary canal, Volume I: Control of food and water intake. Baltimore: Waverly press (American Physiological Society), 1967.

LaBounty, C. E., and Reynolds, G. S. An analysis of response and time matching to reinforcement in concurrent ratio-interval schedules. Journal of the Experimental Analysis of Behavior, 1973, 19, 155-166.

Willis, R. D., Van Hartesveldt, C., Loken, K. K., and Hall, D. C. Motivation in concurrent variable-interval schedules with food and water reinforcers. Journal of the Experimental Analysis of Behavior, 1974, in press.

\section{NOTE}

1. Raw data from Experiment I may be obtained by requesting a copy of "The effects of varying the ratio requirement on a concurrent fixed-ratio: food, fixed-interval: water schedule of reinforcement"- $M$. A. Thesis, March, 1973, by Keith A. Wood, from the Librarian, University of Florida.

(Received for publication July $31,1974$.

\title{
The role of local interactions in behavioral contrast
}

\author{
BEN A. WILLIAMS \\ University of California, San Diego, La Jollo, California 92073
}

\begin{abstract}
Typical behavioral contrast effects were obtained using a multiple variable-interval extinction schedule of reinforcement. Response rate was higher, however, during VI components preceded by a VI component than during a VI component preceded by extinction. This effect, contrary to previous findings concerning sequential effects, challenges recent interpretations of behavioral contrast derived from the literature on autoshaping.
\end{abstract}

Gamzu and Schwartz (1973) have advanced an account of contrast effects in multiple schedules derived

Preparation of the manuscript was supported by NSF Grant GB-42887 to the University of California. from the literature on autoshaping. According to this view, contrast results from the excitation of particular responses, determined by the biology of the organism, simply by virtue of a signal of the transition from periods of low reinforcement probability to periods of 